\title{
Cardiovascular comorbidity in multiple sclerosis patients treated with Mitoxantrone therapy: a cohort study
}

Paolo Ragonese* (1D, Paolo Aridon, Sabrina Realmuto, Giulia Vazzoler, Simona Alessi, Erika Portera, Alessia Bianchi, Fabio Triolo, Maria Antonietta Mazzola, Marco D'Amelio, Giovanni Savettieri and Giuseppe Salemi

\begin{abstract}
Background: Mitoxantrone (MX) has been used as second line therapy for aggressive multiple sclerosis (MS). Potential cardiotoxic effects of MX limit its use; a cumulative dose of up to $100 \mathrm{mg} / \mathrm{m} 2$, has been long considered relatively safe. We calculated the frequency of cardiac side effects in MS patients treated with MX.

Methods: We performed a cohort study including all MS patients treated with MX at the Neurological Department of the University Hospital of Palermo, Italy. Two hundred-sixty-four MS patients diagnosed according to validated criteria were included and followed-up until the end of September 2010. Patients were treated with MX as a second line therapy if they had no previous heart diseases determined by clinical evaluation, electrocardiography, and echocardiography. Treatment administration was made at a monthly dose of $8 \mathrm{mg} / \mathrm{m}^{2}$ for the first three months and at a dose of $12 \mathrm{mg} / \mathrm{m} 2$ every three months. Echocardiography was routinely performed every six months. Treatment was stopped before reaching the final dose if signs had appeared of impaired heart function, confirmed left ventricular ejection fraction reduction lower to $50 \%$, or a confirmed reduction of more than $10 \%$ with respect to the first examination.
\end{abstract}

Results: Heart involvement was observed in $12.4 \%$ of treated individuals, and symptomatic congestive heart failure occurred in $2.7 \%$ of the cohort. A patient had a myocardial infarction, and 3.1\% showed electrocardiographic anomalies not present at baseline.

Conclusion: Our study confirms that cardiac adverse events associated with MX are more common than previously reported.

Keywords: Comorbidity, Multiple sclerosis, Heart failure, Mitoxantrone, Therapy

\section{Background}

Multiple sclerosis (MS) is a chronic disease of the central nervous system due to a damage of myelin sheaths and axons mediated by the immune system and by a neurodegenerative mechanism. MS is one of the major causes of disability in young people. Its causes are still unknown although is generally accepted that MS is a complex autoimmune disorder where genetic and environmental factors both contribute to the pathogenic mechanisms directed against myelin and oligodendrocytes of the central nervous system. Plaques in MS patients are

\footnotetext{
* Correspondence: paolo.ragonese@unipa.it

Department of Experimental Biomedicine and Clinical Neurosciences, University of Palermo, Via Gaetano La Loggia, 1-90129 Palermo, Italy
}

characterized by perivascular infiltrates of mononuclear inflammatory cells, demyelination, axonal loss and gliosis, mainly in the white matter, all along the brain and the spinal cord [1].

Among treatments used for MS, Mitoxantrone (MX) has been used as a second line therapy for multiple sclerosis (MS) [2]. MX is an anthracenedione that reduces lymphocyte proliferation of $\mathrm{B}$ and $\mathrm{T}$ cells, macrophages, and lowers the production of proinflammatory cytokines [3]. Its efficacy in the treatment of MS was tested in clinical trials and, for this reason, the drug in 2000 was approved by FDA $[4,5]$. Cardiotoxic effects of MX limit the cumulative lifetime dose in MS patients to $140 \mathrm{mg} / \mathrm{m}^{2}$. A cumulative dose of no more 
than $100 \mathrm{mg} / \mathrm{m}^{2}$ was considered relatively safe until recently $(2,6)$. More recent observations described higher frequency than expected of cardiac involvement in people exposed to MX [6, 7]. Aim of the present study was to estimate the frequency of heart involvement in a cohort of Sicilian MS patients treated with MX.

\section{Methods}

We used MX at our department after other treatments had failed to demonstrate a reduction of relapse frequency or of disability worsening in patients affected by MS. Before starting MX, patients were first screened for previous cardiovascular diseases by clinical examination, electrocardiography, and by transthoracic echocardiography. We also considered previous uses of cardiotoxic or cytotoxic medications, history of heart disease, current pregnancy or breast-feeding as contraindications to MX treatment. We planned treatment administration at a monthly dose of $8 \mathrm{mg} / \mathrm{m}^{2}$ for the first three months, followed by a dose of $12 \mathrm{mg} / \mathrm{m}^{2}$ every three months up to a cumulative dose of $140 \mathrm{mg} / \mathrm{m}^{2}$. Transthoracic echocardiographs were scheduled every two cycles at the beginning (in the first period when treatment was administered with a monthly regimen), and then every 6 months. These data were recorded in the clinical database of MS patients followed at our Department. Echocardiography was usually performed at the cardiologic department of our University Hospital. If an echocardiography, performed by a patient elsewhere for any reason, showed any kind of changes from baseline, this was evaluated again at our University Hospital cardiologic department. Treatment was stopped before reaching the planned final dose if there were signs of impaired heart function, confirmed left ventricular ejection fraction (LVEF) reduction to less than $50 \%$, or a confirmed reduction of more than $10 \%$ with respect to the first examination. Confirmed modifications of left ventricular ejection fraction (LVEF) had to be present at least in two consecutive evaluations. Further cardiologic evaluations and echocardiographic examinations were also performed six and twelve months after the last administration of MX. Reduction or termination of infusions due to adverse events was left at the physician's judgment. Doses of MX were reduced particularly in case of severe leukopenia, red blood cell or platelet reduction, severe infections or other clinically relevant serological markers modifications. We evaluated the occurrence of several conditions: symptomatic heart failure, changes at electrocardiography or echocardiography suggesting a possible initial heart failure, arrhythmias, valve involvement, pericardial effusion, myocardial infarction. According to the Therapeutics and Technology Assessment Subcommittee of the American Academy of Neurology [2], we considered two different end points: the occurrence of symptomatic congestive heart failure and the frequency of heart involvement (any type) calculated as the rate of patients developing one of the above mentioned conditions. The observation started in 1994 at the time of inclusion of the first patient for MX treatment while for outcome measures we considered September $30^{\text {th }} 2010$ as the end of follow-up. We also compared echocardiographic parameters in the cohort of MS patients at baseline with two hundred individuals studied by echocardiography for other reasons in a similar age group and excluding those who had a previous known heart disease. This group of people included mostly individuals evaluated by echocardiography as a screening procedure to perform agonistic sport activities. We used $t$ test to compare means, and $\mathrm{X}$ square analysis to calculate differences in distribution of LVEF between MS patients and referent subjects at baseline. We also calculated the relative risk (RR) with $95 \%$ confidence intervals (CI) and two-sided $\mathrm{p}$ values for congestive heart failure stratifying by age, gender, cumulative dose of MX, number of MX doses, and disease duration from onset to MX initiation.

The local ethical committee of our University Hospital approved the study, and a consensus was obtained from each patient to be treated with the drug and to be included in our clinical database.

\section{Results}

Patient's characteristics are described in Table 1. Two hundred-sixty-four MS patients (158 women, 106 men) were treated as above described. Mean age at onset of MX treatment was 48 years old (standard deviation 10.3). Mean disease duration was 11.3 years (range 1-38) from onset of symptoms. Sixty-five patients $(24.6 \%)$ had a relapsing-remitting (RR) disease course when they started MX while the remaining 199 (74.8\%) had a secondary or primary progressive disease course with presence of relapses (among them, 21 patients had a primary progressive

Table 1 Demographic and clinical characteristics of MS patients treated with $M X$

\begin{tabular}{ll}
\hline Variable & N \\
\hline Gender (W/M) & $264(158 / 106)$ \\
Mean age at onset (years +/- SD) & $48(10.3)$ \\
Disease course & \\
RR & $65(41 \mathrm{D} / 24 \mathrm{U})$ \\
SP & $132(82 \mathrm{D} / 50 \mathrm{U})$ \\
PP & $38(19 \mathrm{D} / 19 \mathrm{U})$ \\
PR & $29(16 \mathrm{D} / 13 \mathrm{U})$ \\
Mean disease duration (years, range) & $11,3(1-38)$ \\
Mean Follow-up (years +/- SD) & $8(3)$ \\
Mean number of MTX (range) & $7,5(1-21)$ \\
Mean cumulative dose/m2 (range) & $70,8(1-139)$ \\
\hline
\end{tabular}


course). A mean of 7.5 number of MX cycles were administered (range 1-21); mean cumulative dose of MX was 70.8 (range $1-139) \mathrm{mg} / \mathrm{m}^{2}$ of body surface. Two hundred consecutive individuals not affected by MS (106 women, 94 men) were examined as reference group. They had a mean ejection fraction (EF) of 58\%, (median: 60, SD: 5.6). We observed no statistical differences between MS patients and the non-MS patients at baseline. None of these subjects had significant clinical or echocardiographic abnormalities except one who had mild signs of atrial enlargement and mitral valve insufficiency. MS patients had mean a follow-up of 8 years since their inclusion in the study (SD $+/-3)$. Frequency of any type of cardiac function involvement was $12.5 \%$ (33 patients) in MS patients. Signs or symptoms of heart dysfunction occurred within the first two years after the initiation of the treatment in 16 individuals (6\%). In eight patients serious adverse events occurred within the first six months of treatment, including two deaths (determined in one case by severe leukopenia and pneumonia). One patient developed leukaemia while five other patients developed signs of heart dysfunction. Congestive heart failure occurred in $2.7 \%$ of patients ( 3 men, 4 women). A woman died 8 years after MX exposure because of severe heart failure whose onset was attributed to MX treatment (she was considered also among those patients affected by heart failure). Another patient had a myocardial infarction, while 8 (3.1\%) patients showed electrocardiographic abnormalities or arrhythmias not present at baseline. The remaining had a sustained LVEF reduction without evident clinical signs of congestive heart failure. Among other relevant clinical disorders whose onset was considered attributable to MX treatment, we observed three cases of acute leukaemia and a death due to a pancreatic cancer. Two other patients died: one because of a heat shock (after the second MX course) and one of acute pneumonia (after the fifth infusion). Table 2, reports the risk rates for the association between symptomatic heart disease and the considered variables. Risk for heart involvement (any type) was significantly associated with the duration and the dose of the treatment. On the opposite, sex and MS duration since onset did not show any association with the probability to develop a heart disease. We observed an increased risk for

Table 2 Association between MX treatment and the occurrence of symptomatic congestive heart failure

\begin{tabular}{lll}
\hline Variable & RR $(\mathrm{Cl})^{\mathrm{a}}$ & $p$ \\
\hline Age & $3.57(0.98-19.9)$ & 0.12 \\
Gender & $1.05(0.70-2.40)$ & 1 \\
Disease duration & $0.8(0.7-1.1)$ & 0.3 \\
Cumulative dose & $2.3(1.54-3.66)$ & 0.000003 \\
Number of infusion & $2.3(1.4-3.8)$ & 0.0003 \\
\hline
\end{tabular}

${ }_{\mathrm{RR}}=$ relative risk. $\mathrm{Cl}=95 \%$ confidence intervals heart disease related to age at treatment initiation although only close to the statistical significance. Characteristics of patients who developed congestive heart failure are reported in Table 3.

\section{Discussion}

This study confirms previous reports showing frequency of heart disease higher than previously reported in MS patients treated with MX [5-8]. We observed a dose and time related heart toxicity. Rate of heart involvement was also high considering either symptomatic heart failure, subclinical toxicity or measuring any type of heart event. Another recent study performed in France suggested a better safety profile of MX showing [9]. In that study, nearly $5 \%$ of MS patients developed asymptomatic reduction of LVEF at the end of the five-year follow-up (compared to nearly $12 \%$ in our cohort), and just one patient over a cohort of about 800, developed acute heart failure (vs. $2.8 \%$ in the present study). Differences in observed frequencies of MX related heart toxicity might be determined mostly by the methodology used to calculate the occurrence of events (length of follow-up, frequency and number of echocardiography, methods to assess the reduction of LVEF). The inclusion of symptomatic heart failure only, or to encounter LVEF reduction is also determinant of course in the calculation of heart event frequency (i.e. to calculate cardiac disease rate just by including those individuals who present clinical sign of heart failure would produce lower rates compared to studies including as affected by heart disease also those patients with modification of echocardiographic parameters). There is another major difference between the two cohorts represented by the proportion of patients with a RR disease course vs. a progressive course (in our cohort, it approximates the $25 \%$ while in the French cohort was about the 38\%). This is paralleled by the different age at MX initiation which is higher in the Sicilian cohort (mean age of 38 years old for the French study, compare to 48 years for the present cohort). In our cohort, there seems to be a higher risk for heart disease among patients who had MX treatment at an older age although confidence intervals are wide and do not reach the statistical significance. This would also

Table 3 Characteristics of heart involvement in MS patients treated with MTX

\begin{tabular}{lcc}
\hline Type of heart involvement & Percent & Number \\
\hline Heart dysfunction (any type) & 12.5 & 33 \\
Symptomatic heart failure & 2.7 & 7 \\
ECG abnormalities/arrhythmias & 3.1 & 8 \\
Myocardial infarction & 0.4 & 1 \\
Death due to heart failure & 0.4 & 1 \\
Deaths due to other causes (heat shock) & 0.4 & 1 \\
\hline
\end{tabular}


justify the observed higher proportion of heart events in our cohort although the reported risk is not significant, probably because of the low number of events. Other explanations for differences in observed results may depend on the criteria and methodology used in other studies to define and to assess the presence and the severity of heart diseases $[10,11]$. As already mentioned, the distribution of clinical phenotypes of MS patients included in the study, as well as age at initiation of MTX therapy, have to be considered possible factors determining a modification of the observed effects when comparing present results with those from other studies [12]. It has been suggested that in patients undergoing MX treatment for other diseases like cancer, prevention from developing heart failure it is possible to obtain by aerobic exercises, by modification of treatment regimens, or even by routine use of cardiac protective agents or liposomal formulations during the treatment [12]. There is no evidence at present, supporting these suggestions. Moreover, it has not been investigated if these different approaches (reduced dose or time of treatment, or even delayed courses) may warrant a better long-term efficacy particularly among patients like those affected by MS who have a very long expected survival time after exposure to the drug. As previously reported, throughout follow-up time, a small proportion of cases showed at least a partial reversibility of LVEF reduction $[12,13]$. In the present study, anyway, we reported results of sustained LVEF reduction in patients who had a mean follow-up time of eight years and as indicated, the time elapsed from treatment initiation to the onset of congestive heart failure may go over the duration of treatment administration. Another important aspect to consider is that LVEF reduction is only an aspect and an early marker of cardiomyopathy which is a more complex pathologic process characterized by a global systolic dysfunction when left ventricular enlargement is still not clearly evident $[12,14]$. This is of outstanding importance because other reports have shown that cardiomyopathy in patients treated with MX can occur early after few infusions when either clinical symptoms nor LVEF reduction may be already detected [13-16]. As previously described, cardiomyopathy may in fact occur early, manifesting with aspecific ST-segment and T-wave abnormalities, or as the late dose-related heart failure and left ventricular dysfunction $[15,17]$. In our study and in previous observations [2], myocardial infarctions and arrhythmias had also been observed and included in the calculation of MX related toxicity. For these reasons we believe that early signs must also be accurately searched, as treated patients could later develop a congestive heart failure and also because recent studies indicate the possibility of an efficacious early treatment $[15,16,18,19]$.

Few studies have been performed in Europe reporting the incidence of heart dysfunction in the general population with a similar age distribution $[17,20]$. Two studies performed in Italy investigating the frequency of heart dysfunction in two cohorts of individuals who performed cardiologic evaluations to monitor heart function in young adult individuals [18-23] showed rates of hypertrophic cardiomyopathy between 0.07 and $0.7 \%$. In the group of individuals performing echocardiography and not affected by MS we considered for comparisons, we did not observed any difference at baseline with respect to MS patients and, as previously reported, only one had significant signs of potential heart disease at echocardiographic examination. A limitation relies anyway on the fact that we could not obtain a follow-up examination of referent subjects as those people were screened only once. Nevertheless, we did not observed in our MS cohort at baseline any significant heart disease sign nor significant abnormalities were present at echocardiography performed before MTX treatment, since this would have represented an exclusion criteria for this specific treatment. Finally, we observed several serious side effects like cancers; in our cohort, we observed in fact three case of leukaemia, one lung cancer, one gut cancer, and one of the pancreas. This confirms the need for a strict control of MX related toxicity even after treatment stopping.

\section{Conclusion}

Although the use of MTX in patients with MS has declined in last years, it still represents an alternative in selected cases with very aggressive disease. Furthermore, the debate is still open about the opportunity to adopt an induction strategy able to exert effects on innate immunity, when approaching MS treatment and in this perspective, other drugs have still to prove to be as effective as MTX could be. The present study suggest a significantly increased risk for cardiovascular comorbidity and toxicity among MS patients treated with MTX. Heart involvement seemed to be dependent on the dose and the duration of MTX exposure but not on age or disease duration. On the opposite, in the cohort of MS patients included there were no differences in echocardiographic parameters at baseline with respect to another group of individuals not affected by MS. Although the results of the present study confirm previous concerns about a broad and prolonged use of MTX for the treatment of MS, some additional consideration are needed. It has already been suggested that, in the effort to guarantee MS patients with the best possible treatment to prevent the accrual of not reversible disability, particularly for patients with clinical subtypes that do not have yet valid therapeutic opportunities, MTX could still represent one the few chances available [24]. What it is still open to debate is how to use this drug. A reasonable approach might consider a short- 
term induction strategy with lower dosages at each administration and low cumulative exposure. Based on this study, this could not be still sufficient to avoid toxic effects, due to the possibility of an early toxicity. For this reason, this therapy should be considered only by trying to minimize risk for cardiotoxicity together with an active strict monitoring strategy of treated individuals. At present, this may be achieved by using personalized therapeutic approaches using pharmacogenetics markers [25] or by the use of analogues of MTX, like the Pixantrone, which has been proposed as an alternative possible therapy for MS patients with an active disease not responding to current treatments [26].

\section{Acknowledgements}

Not applicable.

\section{Funding}

The present study was not supported by any funding.

\section{Availability of data and materials}

The datasets used and/or analyzed during the current study available from the corresponding author on reasonable request.

\section{Authors' contributions}

$P R, G S$, and GS planned the study, revised the database contributed to the analyses, discussed the results, drafted and revised the manuscript. SR, GV, $S A, E P, A B$, contributed to selection of patients, building up of the database and retrieving all necessary information, revising the manuscript. PA, GV, MAM, and MD'A contributed to revising the database, performing analyses, discussing the results, revising the manuscript. All authors read and approved the final manuscript.

\section{Competing interests}

The authors report no competing interest with respect to this study.

\section{Consent for publication}

Not applicable.

\section{Ethics approval and consent to participate}

The local ethical committee of our University Hospital approved the study and a consensus was asked to each patient to be treated with the considered drug and to be included in our clinical database.

\section{Publisher's Note}

Springer Nature remains neutral with regard to jurisdictional claims in published maps and institutional affiliations.

Received: 21 January 2017 Accepted: 10 May 2017

Published online: 24 July 2017

\section{References}

1. Compston ACC, Lassmann H, McDonald I, Miller D, Noseworthy J, Smith K, Wekerle H, editors. McAlpine's Multiple Sclerosis. 4th ed. Philadelphia, PA: Churchill Livingstone Elsevier; 2004

2. Marriott JJ, Miyasaki JM, Gronseth G, O'Connor PW. Evidence report: the efficacy and safety of mitoxantrone (novantrone) in the treatment of multiple sclerosis: report of the therapeutics and technology assessment subcommittee of the American academy of neurology. Neurology. 2010;74:1463-70.

3. Ehninger G, Schuler U, Proksch B, Zeller KP, Blanz J. Pharmacokinetics and metabolism of mitoxantrone. A review. Clin Pharmacokinet. 1990;18:365-80.

4. Martinelli Boneschi F, Vacchi L, Rovaris $M$, et al. Mitoxantrone for multiple sclerosis. Cochrane Database Syst Rev. 2013;31(5):CD002127.

5. Hartung HP, Gonsette R, Ko "nig N, et al. A placebo-controlled, double-blind, randomised, multicentre trial of mitoxantrone in progressive multiple sclerosis. Lancet. 2002;360:2018-25.
6. Kingwell E, Koch M, Leung B, et al. Cardiotoxicity and other adverse events associated with mitoxantrone treatment for MS. Neurology. 2010;74:1822-6.

7. Ghalie RG, Edan G, Laurent M, et al. Cardiac adverse effects associated with mitoxantrone (Novantrone) therapy in patients with MS. Neurology. 2002:59:909-13.

8. Edan G, Miller D, Clanet M, et al. Therapeutic effect of mitoxantrone combined with methylprednisolone in multiple sclerosis: a randomised multicentre study of active disease using MRI and clinical criteria. J Neurol Neurosurg Psychiatry. 1997;62:112-8.

9. Le Page E, Leray E, Edan G, French Mitoxantrone Safety Group. Long-term safety profile of mitoxantrone in a French cohort of 802 multiple sclerosis patients: a 5-year prospective study. Mult Scler. 2011;7:867-75.

10. Goffette S, van Pesch V, Vanoverschelde JL, Morandini E, Sindic CJ. Severe delayed heart failure in three multiple sclerosis patients previously treated with mitoxantrone. J Neurol. 2005:252:1217-22.

11. Pattoneri P, Sozzi F, Pelà G. Assessment of mitoxantrone-induced cardiotoxicity in patients with multiple sclerosis: a tissue Doppler echocardiographic analysis. Echocardiography. 2009;26:397-402.

12. Fleischer V, Salmen A, Kollar S, et al. Cardiotoxicity of mitoxantrone treatment in a german cohort of 639 multiple sclerosis patients. J Clin Neurol. 2014;10:289-95.

13. Bernitsas E, Wei W, Mikol DD. Suppression of mitoxantrone cardiotoxicity in multiple sclerosis patients by dexrazoxane. Ann Neurol. 2006;59:206-9.

14. Smith LA, Cornelius VR, Plummer CJ, et al. Cardiotoxicity of anthracycline agents for the treatment of cancer: Systematic review and meta-analysis of randomized controlled trials. BMC Cancer. 2010:10:337.

15. Khatri BO, Wroblewski M, Kramer J, Dukic M, Poplar A, Anderson AJ. Mitoxantrone in worsening secondary progressive multiple sclerosis: a prospective, open-label study. Curr Ther Res. 2006;67:55-65.

16. Keefe DL. Anthracycline-induced cardiomyopathy. Semin Oncol. 2001;28:2-7.

17. Avasarala JR, Cross AH, Clifford DB, Singer BA, Siegel BA, Abbey EE. Rapid onset mitoxantrone-induced cardiotoxicity in secondary progressive multiple sclerosis. Mult Scler. 2003:9:59-62.

18. Paul F, Dörr J, Würfel J, Vogel HP, Zipp F. Early mitoxantrone-induced cardiotoxicity in secondary progressive multiple sclerosis. J Neurol Neurosurg Psychiatry. 2007;78:198-200.

19. Yeh ETH, Tong AT, Lenihan DJ, et al. Cardiovascular complications of cancer therapy: diagnosis, pathogenesis, and management. Circulation. 2004; 109:3122-31.

20. Cardinale D, Colombo A, Lamantia G, et al. Anthracycline-induced cardiomyopathy. Clinical relevance and response to pharmacologic therapy J Am Coll Cardiol. 2010;55:213-20.

21. Mosterd A, Hoes AW. Clinical epidemiology of heart failure. Heart. 2007:93:1137-46.

22. Corrado D, Basso C, Schiavon M, Pelliccia A, Thiene G. Pre-participation screening of young competitive athletes for prevention of sudden cardiac death. J Am Coll Cardiol. 2008;52:1981-9.

23. Corrado D, Basso C, Schiavon M, Thiene S. Screening for hypertrophic cardiomyopathy in young athletes. N Engl J Med. 1998;339:364-9.

24. Cocco E, Marrosu M. The current role of mitoxantrone in the treatment of multiple sclerosis. Expert Rev Neurother. 2014;14:607-16.

25. Cotte S, von Ahsen N, Krus N. ABC-transporter gene-polymorphisms are potential pharmacogenetic markers for mitoxantrone response in multiple sclerosis. Brain. 2009:132:2517-30.

26. Gonsette R, Debouverie M, Sindic C, Ferré JC, Edan G. Pixantrone: a B-cell-depleting immunosuppressant for multiple sclerosis patients with active disease. Mult Scler. 2016:6:817-21. 